\title{
Structure-material Integrated Design for a Spacecraft Rib
}

\author{
Zijie Chen, Ziling Chen, Huang Li, Jie Liu* \\ School of Mechanical and Electric Engineering, Guangzhou University, Guangzhou, P. R. China \\ Email address: \\ 925990446@qq.com (Zijie Chen),2320882071@qq.com (Ziling Chen), 1711803207@qq.com (Huang Li), jliu@gzhu.edu.cn (Jie Liu) \\ ${ }^{*}$ Corresponding author
}

\section{To cite this article:}

Zijie Chen, Ziling Chen, Huang Li, Jie Liu. Structure-material Integrated Design for a Spacecraft Rib. American Journal of Mechanical and Materials Engineering. Vol. 4, No. 4, 2020, pp. 81-88. doi: 10.11648/j.ajmme.20200404.11

Received: February 20, 2021; Accepted: February 26, 2021; Published: March 4, 2021

\begin{abstract}
It is the goal that the aerospace industry has been continuously pursuing to meet the lightweight design with excellent mechanical properties. A structure-material integrated design framework is proposed to enhance the load-bearing rate of a spacecraft rib significantly, based on the optimization design theory. The structure-material integrated design framework is realized in two steps by commercial software Altair Solidthinking Inspire. The first step is that topology optimization is performed to a spacecraft rib at the macroscopic scale, with the minimum mass and the constraints of the additive manufacturing process and stress; while the second step is to optimally infill the lattice structure at the microscopic scale by minimizing the mass and constraining the additive manufacturing process and stress. Representative samples for the optimal rib structure are then fabricated by the additive manufacturing technique, and the tensile test is finally carried out to obtained the load-bearing rate for the different samples. The results show that the spacecraft rib's load-bearing rate is increased by $122.73 \%$ by the proposed structure-material integrated design framework compared to the traditional one; moreover, it is significantly more efficient than the direct topology optimization and lattice optimization design. The structure-material integrated design framework shown in this study can provide an efficient way to aerospace structures with lightweight and superior mechanical properties.
\end{abstract}

Keywords: Spacecraft Rib, Structure-Material Integrated Design Framework, Load-Bearing Rate, Topology Optimization, Lattice Optimization Design

\section{Introduction}

Since aerospace equipment's weight is directly related to energy consumption and endurance, the lightweight design has always been an eternal pursuit. Besides, the operating environment of aerospace equipment is often very harsh, so the high mechanical properties of key structures are also the pursuit goals of this field to meet its high-reliability service requirements. Among them, the ratio of the bearing capacity to its quality, namely the load-bearing rate, is one of the critical evaluation indicators and is also the focus of this work.

Continuum topology optimization method provides an effective means for designing aerospace equipment structures with high load-bearing ratio [1]. Topology optimization refers to the process of achieving the best structural performance by reducing or adding materials under specific boundary conditions and loads and meeting specified constraints. This method has developed rapidly in the past thirty years, and forms such as the homogenization method [2], the solid isotropic material with penalization (SIMP) method [3], the evolutionary structural optimization (ESO) method [4, 5], level set methods [6], bubble method [7], moving morphable component method [8]. In addition, taking the rapid development of additive manufacturing as an opportunity, topology optimization has gradually played a vital role in the aerospace field in recent years. For example, the $\mathrm{Su}-57$ recently launched a topology optimization design program, whose properties in terms of thrust-to-weight ratio, manoeuvrability, and super patrol, have been greatly improved [9]. Besides, the porous material structure represented by the lattice structure has excellent properties such as high specific modulus, high specific stiffness, adequate energy absorption capacity, good heat dissipation ability, etc. [10-15]. Comprehensively considering the characteristics of the aforementioned topology optimization technology and the porous material structures, the structure-material integrated design method is given birth to [16-19]. The method has dramatically exerted the potential of 
materials and has excellent application prospects in aerospace lightweight structure design.

On the macro scale, Remouchamps et al. [20] proposed a two-layer optimization scheme that combines topology optimization and geometric optimization and applied it to aircraft hangers' lightweight design. Wong et al. [21] optimized the design of aircraft landing gear components based on considering the dynamic load. Felix et al. [22] proposed a topology optimization method considering the weight of the structure and applied it to design the wing's internal structure. In terms of the structure-material integrated design, Liu et al. [23] designed an aircraft spoiler infilled by 3D Kagome lattice sandwich structure with high specific stiffness. Wu et al. [24] proposed a novel method to optimize the outer shell and the inner filling porous simultaneously. $\mathrm{Yu}$ et al. [25] conducted a topology optimization design for a structure with a shell on the outer layer and a filled lattice, which effectively alleviated the stress concentration phenomenon. Qiu et al. [26] proposed a structure-material integrated design approach in the ESO framework. However, most of the existing researches are limited to the pursuit of structural rigidity maximization under volume constraints, and there are few researches on engineering issues such as additive manufacturing processes and maximum structural tensile strength ratio under stress constraints.

The study aims to use optimization methods to improve its load-bearing rate of a specific spacecraft rib. The spacecraft ribs are in a stretched state under regular operation, so the optimization goal is the tensile strength ratio. Based on optimization design theory, the commercial software Altair Solidthinking Inspire is used, and the structure-material integrated design is realized in two steps. The first step is to use the additive manufacturing process and stress as constraints to conduct the minimum mass topology optimization design of the spacecraft rib; the second step is to carry out the lattice structure optimization by pursuing the minimum mass, and with the additive manufacturing process and stress as the constraints. Finally, the optimal rib structure samples are fabricated by 3D printing, and the tensile tests are conducted to quantify the load-bearing rate of the optimized spacecraft rib. The paper is organized as follows. After the introduction, Section 2 describes the structure-material integrated design theory. Section 3 shows the design of the spacecraft rib. Experimental studies are performed in Section 4. The manuscript is closed with conclusions in Section 5.

\section{The Structure-Material Integrated Design Theory}

\subsection{Topology Optimization of the Stress-Constrained Mass Minimum}

The variable density method represented by the SIMP method is currently the most used and most mature topology optimization method [3]. The design domain is divided into elements, and the design variable is the density of each element. Based on SIMP model, Young's modulus can be expressed as,

$$
E_{i}=x_{i}^{p} E_{0}
$$

where $E_{i}$ is Young's modulus of the $i$-th element; $x_{i}$ is the relative density of the $i$-th element; $p$ is the penalty factor, used to punish the intermediate density, generally $p=3 ; E_{0}$ is Young's modulus of the solid element.

For the topology optimization of stress-constrained mass minimum problem, the mathematical model can be expressed as,

$$
\begin{array}{ll}
\text { find } & \mathrm{X}=\left\{x_{1}, x_{2}, x_{3}, \ldots x_{i}\right\}^{\mathrm{T}} \\
\min & V(\mathrm{X})=\sum_{i=1}^{N} V\left(x_{i}\right) \\
\text { s.t. } & \sigma\left(x_{i}\right) \leq[\sigma] \\
& \mathrm{KU}=\mathrm{F} \\
& 0<x_{\min } \leq x_{i} \leq 1 \quad(i=1, \ldots, N)
\end{array}
$$

where $V$ is the total volume of the structure; $V\left(x_{i}\right)$ is the volume of the $i$-th element; $N$ is the total number of elements; $\sigma\left(x_{i}\right)$ is the stress at the center of the $i$-th element; $[\sigma]$ is the allowable stress; $\boldsymbol{K}$ is the global stiffness matrix; $\boldsymbol{U}$ is the global Displacement array; $\boldsymbol{F}$ is the load array; $x_{\min }$ is the minimum relative density, and its value is slightly greater than 0 to avoid the singularity of the stiffness matrix.

For solving (2), the finite element (FE) method is used to calculate the objective function and sensitivity information at each iteration. The optimization criterion method or the method of moving asymptotes [27-30], combined with the sensitivity information to update the design variables until the convergence condition is met. Through the topological optimization design of the minimum mass under stress constraints, the material in the non-critical areas of the spacecraft rib can be reduced, and its load-bearing rate can be improved to a certain extent.

\subsection{Lattice Optimization of the Stress-Constrained Mass Minimum}

The lattice structure is employed to infill the design area, and the cross-sectional area of the bars in the lattice structure is used as the design variable to obtain the optimal structure driven by mechanical properties. Lattice optimization of stress-constrained mass minimum problem can be defined as,

$$
\begin{array}{ll}
\text { find } & \mathrm{A}=\left\{A_{1}, A_{2}, A_{3}, \ldots A_{i}\right\}^{\mathrm{T}} \\
\min & V(\mathrm{~A})=\sum_{i=1}^{N_{l}} l A_{i} \\
\text { s.t. } & \left|\sigma_{i} A_{i}\right| \leq[\sigma]_{l} A_{i} \\
& \mathrm{KU}=\mathrm{F} \\
& 0<A_{L} \leq A_{i} \leq A_{U} \quad\left(i=1, \ldots, N_{l}\right)
\end{array}
$$

where $A_{i}$ is the cross-sectional area of the $i$-th bar; $l$ is the 
length of the bar; $N_{l}$ is the total number of bars; $\sigma_{i}$ is the axial stress of the $i$-th bar cross-section; $[\sigma]_{l}$ is the allowable stress of the bar; $A_{L}$ and $A_{U}$ are the minimum and the maximum value of the cross-sectional area of the bar, respectively.

In lattice optimization, the design area is first infilled with lattice structures, and the responses of the objective and constraints are calculated using the FE method. The sensitivity analysis is conducted to update the bar design variables until the convergence conditions are satisfied. Through the lattice optimization, the non-critical area materials of the optimized structure obtained from the macro-scale topology

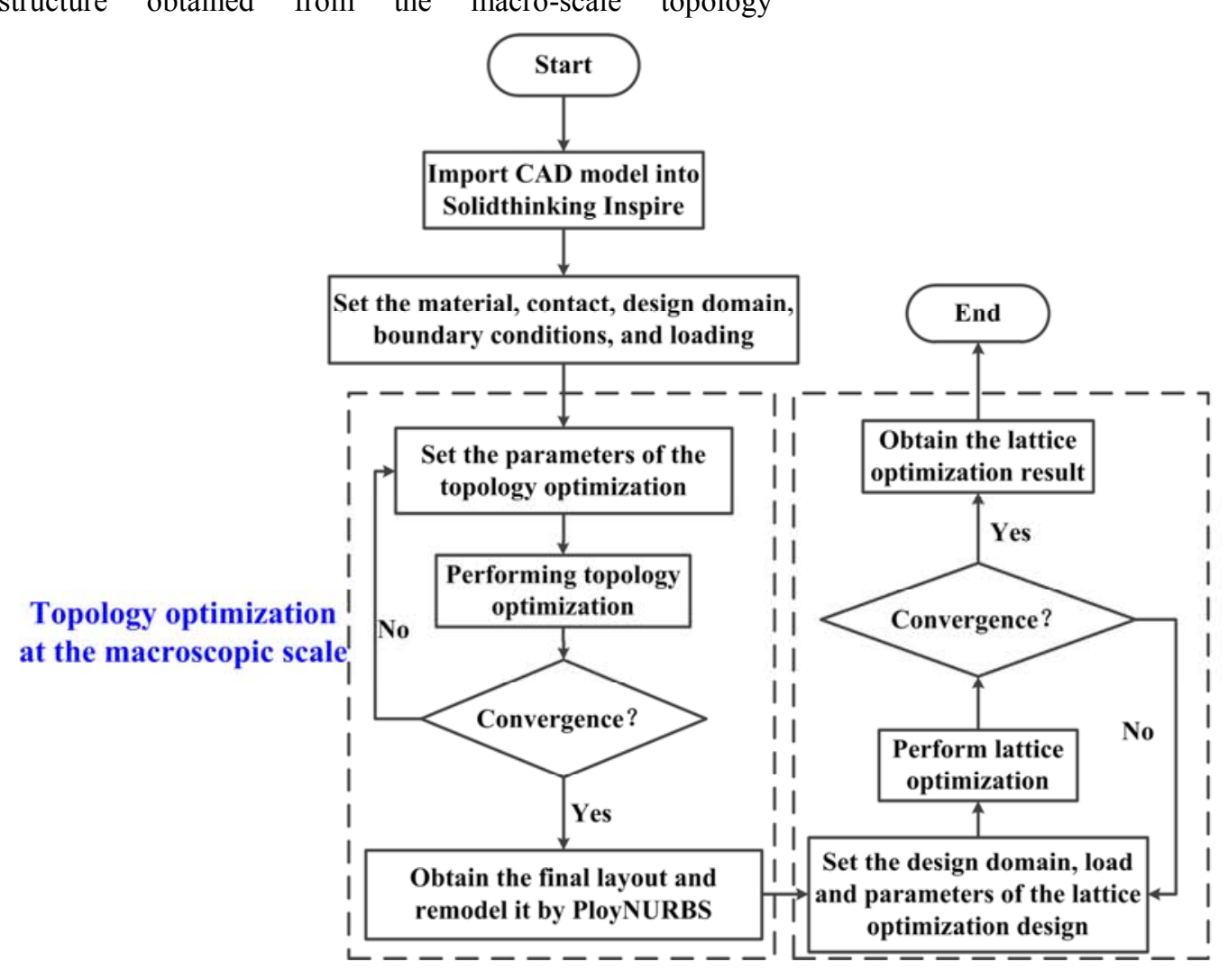

Figure 1. Optimization flow chart. optimization are replaced with the lattice structures, further significantly improving the load-bearing rate.

\section{Structure-Material Integrated Design of a Spacecraft Rib}

Altair Solidthinking Inspire software is employed to optimize a spacecraft rib based on the aforementioned optimization design theory. The optimization flowchart is shown in Figure 1.

\subsection{Design Problem}

The thickness of the main body and both ends of a spacecraft ribs are $6 \mathrm{~mm}$ and $2 \mathrm{~mm}$, respectively. The other geometric parameters are shown in Figure 2(a), and the unit is $\mathrm{mm}$. The upper part and the lower part are respectively provided with 4 and 3 through holes to connect with the fixture through pins. The upper and lower fixtures are subjected to loads in opposite directions and equal in magnitude, as shown in Figure 2(b). Due to the precision limit of the $3 \mathrm{D}$ printer, the additive manufacturing constraint that neither the wall thickness nor the bar diameter is less than $0.5 \mathrm{~mm}$ must be met during the optimization design process. The optimized structure sample was manufactured by a light-curing 3D printer using photosensitive resin, and Young's modulus, Poisson's ratio, density and tensile strength of the material were $1.1 \mathrm{GPa}, 0.4,1.18 \mathrm{~g} / \mathrm{cm}^{3}$ and $23 \mathrm{MPa}$, respectively.

\subsection{Macro-scale Topology Optimization Design}

Considering the additive manufacturing process and installation and assembly requirements with the minimum wall thickness of the structure not less than $0.5 \mathrm{~mm}$, the initial design domain is divided into two parts, i.e. design domain and non-design domain, shown in Figure 3(a). Metal materials are used for fixtures and pins, and Young's modulus, Poisson's ratio, density and yield stress are $195 \mathrm{GPa}, 0.29,8 \mathrm{~g} / \mathrm{cm}^{3}$ and $215 \mathrm{MPa}$, respectively; the aforementioned photosensitive resin materials are used for the spacecraft rib. To enhance the connectivity of the optimized structure at the non-stretched end, fixed constraints are added to the contact surface between the two ends of the spacecraft and the middle part, as shown in Figure 3(b). The final layout of the spacecraft rib is shown in Figure 3(c). Furthermore, the PloyNURBS geometric reconstruction is carried out, as depicted in Figure 3(d), further applied to the lattice optimization in the succeeding 
paragraph.

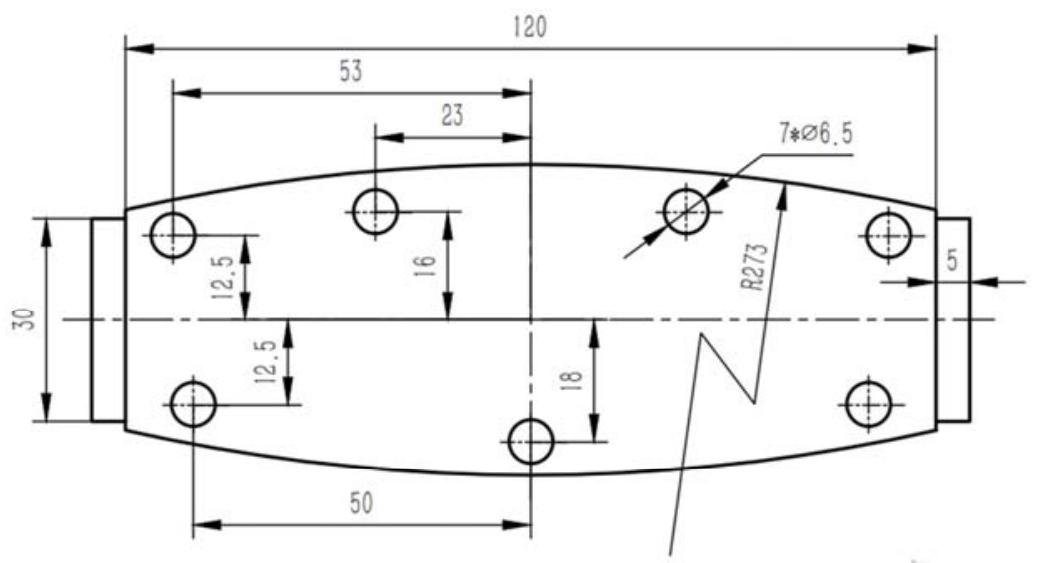

(a)

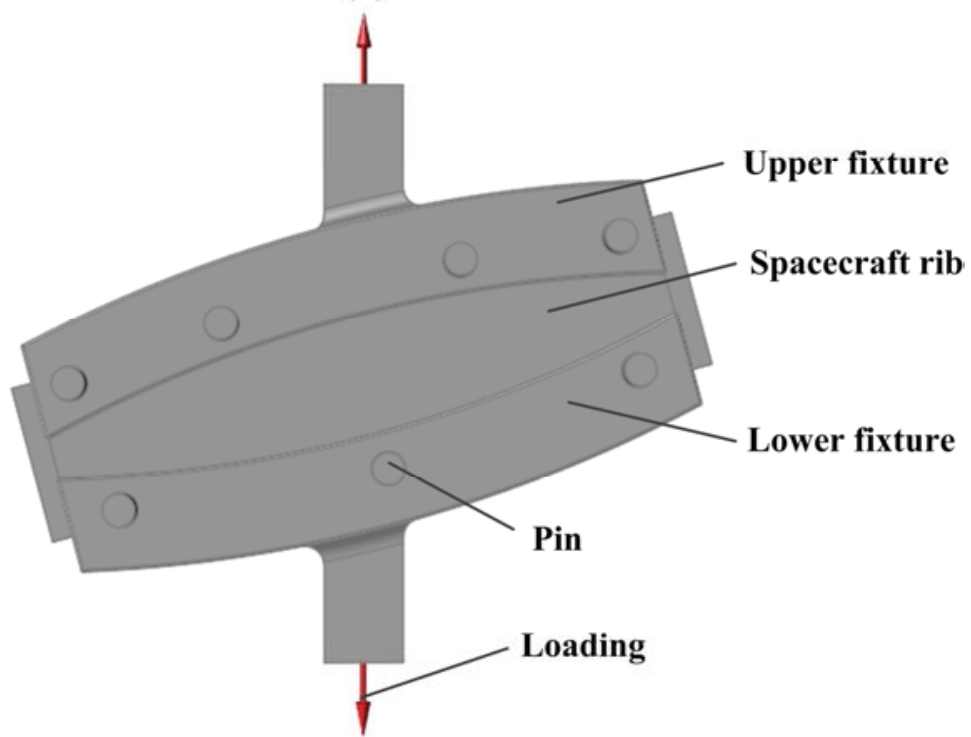

(b)

Figure 2. Schematic diagram of a spacecraft rib: (a) geometric parameter; (b) boundary and loading conditions.

(a)

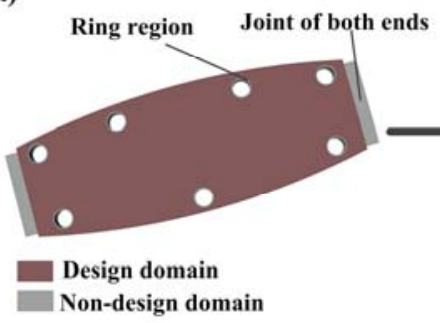

(d)

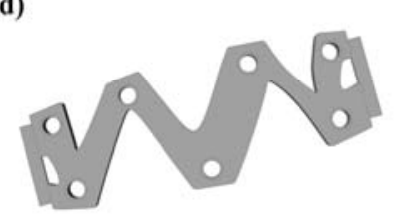

(b)

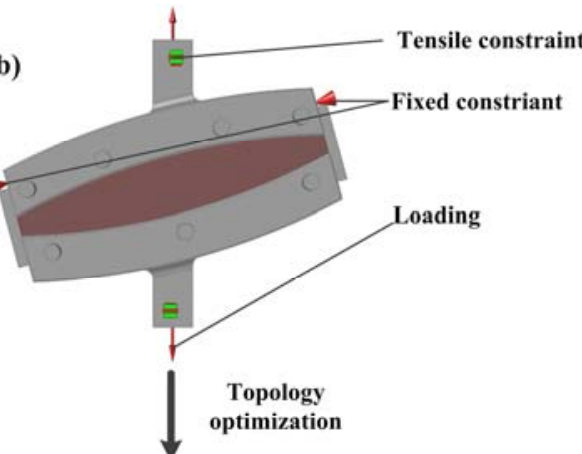

(c)

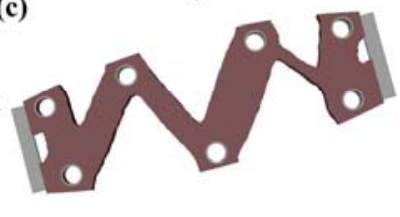

Figure 3. Topology optimization design at the macro scale: (a) design domain and non-design domain; (b) boundary and loading conditions; (c) final layout; (d) reconstructing the CAD model. 


\subsection{Optimal Design of Micro-scale Lattice Structure}

Set the non-critical area of the final layout obtained by the topology optimization as the design domain of lattice optimization, as shown in Figure 4(d). Except for the fixed constraints imposed by considering the connectivity of the non-stretched ends of the structure, the material properties and other boundary conditions of the lattice optimization are the identical to those at the macro scale, as shown in Figure 4(e). The length of the bar is $5 \mathrm{~mm}$, and a constraint is posed to ensure the bar's diameter is not less than $0.5 \mathrm{~mm}$, i.e. between $0.5 \mathrm{~mm}$ and $1.5 \mathrm{~mm}$. The final layout obtained from the lattice optimization shown in Figure 4(f).

For ease of description, the original spacecraft rib, the final layout obtained by topology optimization, and final layout obtained by the structure-material integrated design framework are called the original structure (OS), topology optimized structure (TOS) and topology-lattice (non-critical area) optimized structure (TLNOS), respectively. In addition, based on the topology optimization design, two other structures are produced, namely, lattice optimization of the overall structure and direct infilling the non-critical areas with lattice structures. They are called topology-lattice optimized structure (TLOS) and topology-lattice (non-critical area) infilling structure (TLNIS), respectively, which will be comparatively studied in experimental studies. (a)

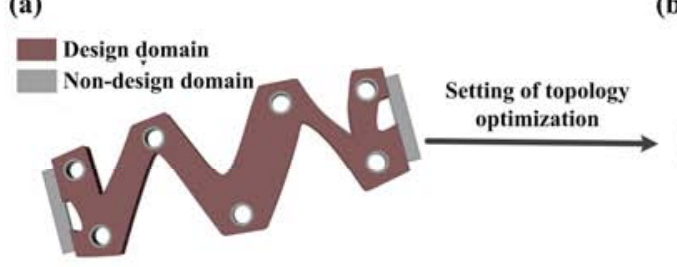

(b)

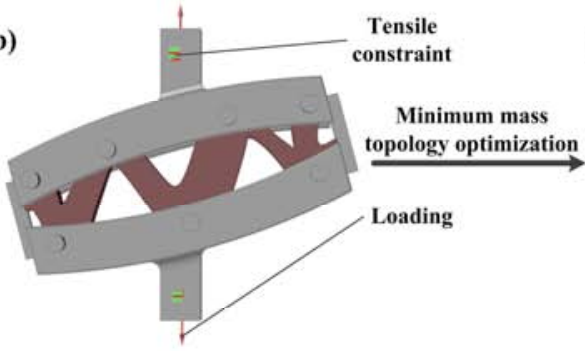

(c)

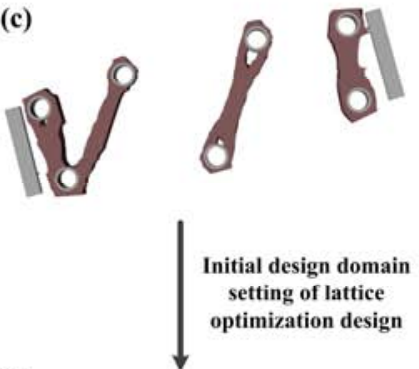

(e)

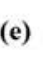

(f)
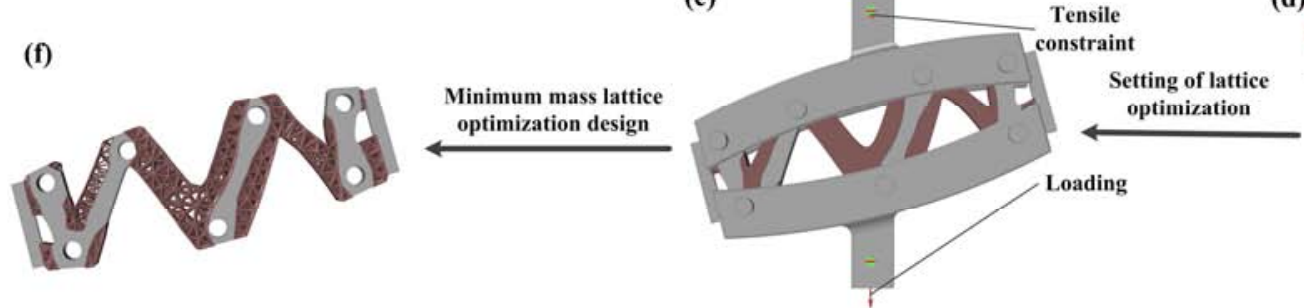

(d)

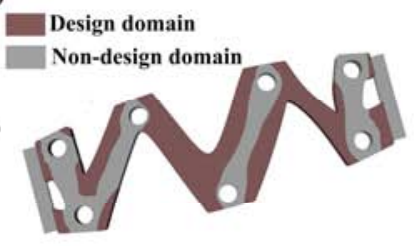

Figure 4. Lattice optimization design at micro scale: (a) design domain and non-design domain of topology optimization; (b) boundary conditions and loading of topology optimization; (c) final layout from topology optimization; (d) design domain and non-design domain of lattice optimization; (e) boundary conditions and loading of lattice optimization; (f) final layout from lattice optimization.

\section{Experimental Study}

Utilizing a light-curing 3D printer (Formlabs Form2) and an electronic tensile testing machine (MTSCMT4103), the above five structural samples were fabricated by an additive manufacturing method. The tensile force-displacement response curve is obtained through the tensile test to determine the maximum load, $\mathrm{F}$, of each structure, further obtaining the load-bearing rate, $R=F / m$, where $\mathrm{m}$ is the structural mass. The tensile test setup is shown in Figure 5(f), where samples for the five structures are depicted in Figure 5(a) (e). Three sets of tests are performed on each structure, and the force-displacement curve obtained is shown in Figure 6. The mass of each structure and the corresponding load-bearing rate are shown in Table 1. In order to achieve a more intuitive comparison effect, Figure 7 also gives the load-bearing rate histogram.

It can be seen from Table 1 and Figure 7 that the average loading-bearing rates of the five structures are $33.30 \mathrm{~N} / \mathrm{g}$, $60.93 \mathrm{~N} / \mathrm{g}, 74.17 \mathrm{~N} / \mathrm{g}, 35.89 \mathrm{~N} / \mathrm{g}$ and $71.34 \mathrm{~N} / \mathrm{g}$, respectively.
Compared with the OS, the load-bearing rate of TOS is increased by $82.97 \%$; compared to the TOS, the load-bearing rate of TLNOS is enlarged by $21.73 \%$. Therefore, the proposed structure-material integrated design framework enhances the spacecraft rib's load-bearing rate by $122.73 \%$. It shows that both the topology optimization and lattice optimization can improve the spacecraft rib's load-bearing rate to a certain extent, and the proposed design framework has a significantly better effect. Besides, compared to OS, the load-bearing rate of TLOS has been increased by $7.78 \%$, while the load-bearing rate of the TLNIS has increased by $114.23 \%$, which is less than $122.73 \%$, indicating that it can improve the load-bearing ratio of the ribs but has a relatively weak effect than the proposed design framework, and the lattice optimization of the whole structure directly cannot improve the tensile strength ratio. In the existing research, the lattice structure is mainly to improve the buckling strength ratio of the structure [31], which further illustrates the importance of proposing a structure-material integrated design framework that can increase the tensile strength ratio of the spacecraft rib. 


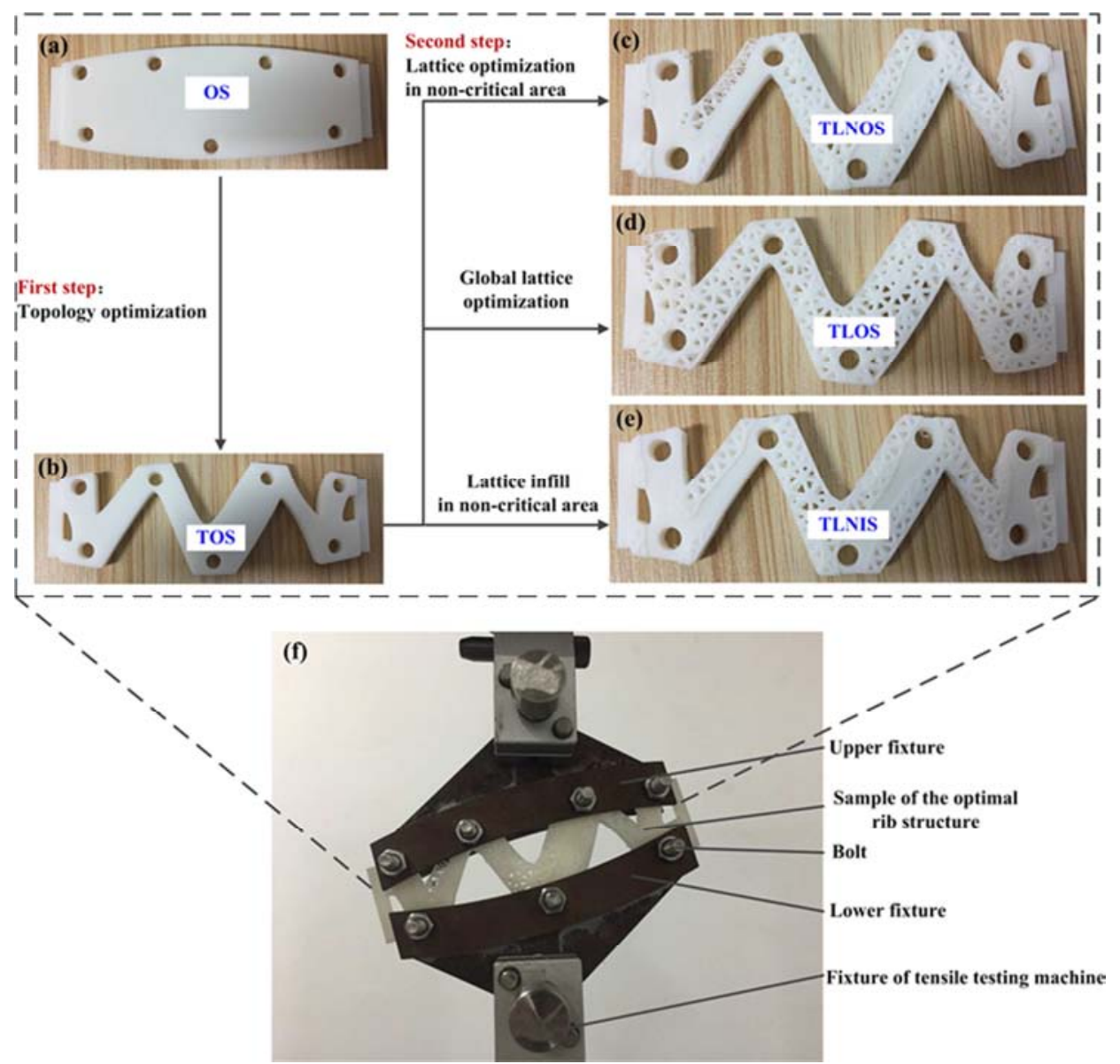

Figure 5. (a e) Representative samples for the optimal rib structure: (a) OS; (b) TOS; (c) TLNOS; (d) TLOS; (e) TLNIS; and (f) tensile test setup.

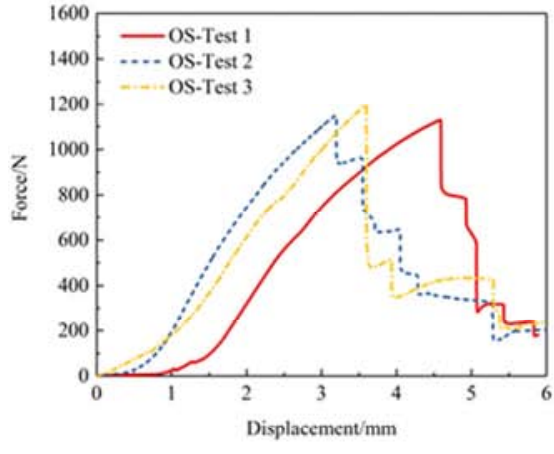

(a)

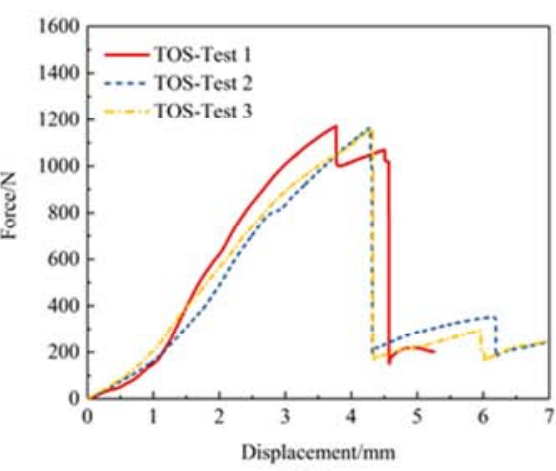

(b)

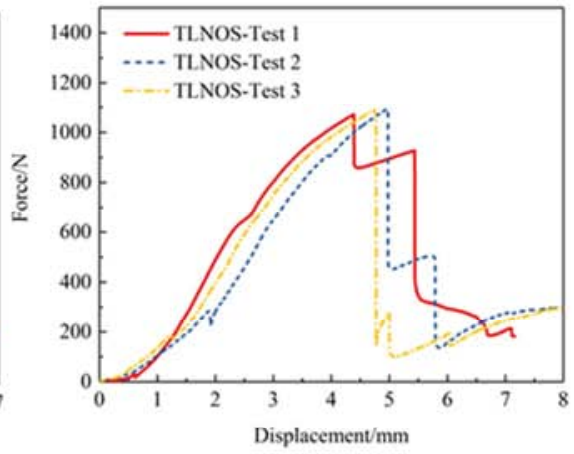

(c)

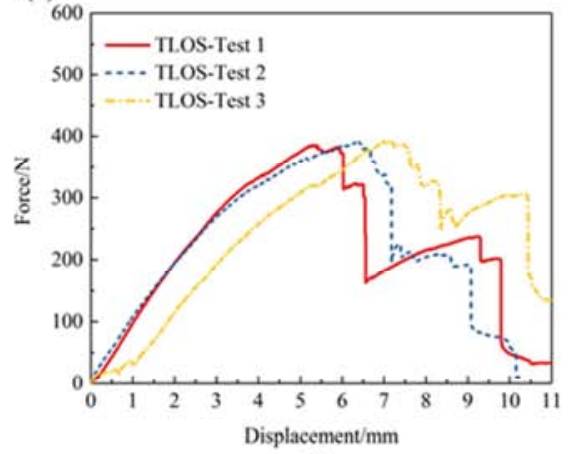

(d)

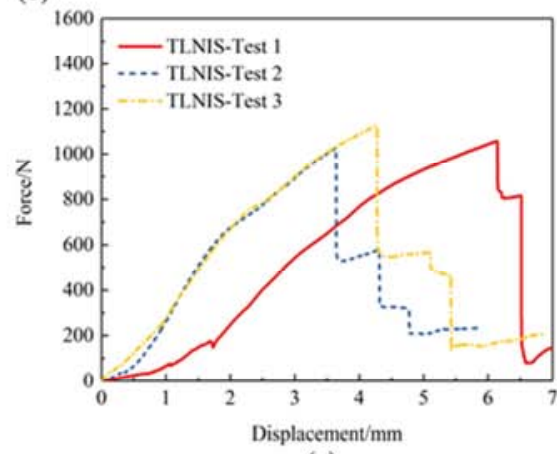

(e)

Figure 6. Force-displacement curve of tensile tests: (a) OS; (b) TOS; (c) TLNOS; (d) TLOS; (e) TLNIS. 
Table 1. Tensile test results of different samples.

\begin{tabular}{|c|c|c|c|}
\hline OS & Group 1 & Group 2 & Group 3 \\
\hline Mass $(\mathrm{m} / \mathrm{g})$ & 34.91 & 34.73 & 34.86 \\
\hline Maximum load $(F / \mathrm{N})$ & 1129 & 1152 & 1199 \\
\hline Load-bearing rate $(R /(\mathrm{N} / \mathrm{g}))$ & 32.34 & 33.17 & 34.39 \\
\hline Average loading-bearing rate $\left(R_{a}(\mathrm{~N} / \mathrm{g})\right)$ & 33.3 & & \\
\hline TOS & Group 1 & Group 2 & Group 3 \\
\hline Mass $(\mathrm{m} / \mathrm{g})$ & 19.18 & 19.15 & 19.06 \\
\hline Maximum load $(F / \mathrm{N})$ & 1171 & 1165 & 1161 \\
\hline Load-bearing rate $(R /(\mathrm{N} / \mathrm{g}))$ & 61.05 & 60.84 & 60.91 \\
\hline Average loading-bearing rate $\left(R_{a}(\mathrm{~N} / \mathrm{g})\right)$ & 60.93 & & \\
\hline TLNOS & Group 1 & Group 2 & Group 3 \\
\hline $\operatorname{Mass}(m / g)$ & 14.32 & 14.89 & 14.79 \\
\hline Maximum load $(F / \mathrm{N})$ & 1072 & 1097 & 1094 \\
\hline Load-bearing rate $(R /(\mathrm{N} / \mathrm{g}))$ & 74.86 & 73.67 & 73.97 \\
\hline Average loading-bearing rate $\left(R_{a}(\mathrm{~N} / \mathrm{g})\right)$ & 74.17 & & \\
\hline TLOS & Group 1 & Group 2 & Group 3 \\
\hline $\operatorname{Mass}(m / g)$ & 10.69 & 10.5 & 11.45 \\
\hline Maximum load $(F / \mathrm{N})$ & 386 & 391 & 393 \\
\hline Load-bearing rate $(R /(\mathrm{N} / \mathrm{g}))$ & 36.11 & 37.24 & 34.32 \\
\hline Average loading-bearing rate $\left(R_{a}(\mathrm{~N} / \mathrm{g})\right)$ & 35.89 & & \\
\hline TLNIS & Group 1 & Group 2 & Group 3 \\
\hline Mass $(\mathrm{m} / \mathrm{g})$ & 14.93 & 15 & 15.06 \\
\hline Maximum load $(F / \mathrm{N})$ & 1058 & 1027 & 1125 \\
\hline Load-bearing rate $(R /(\mathrm{N} / \mathrm{g}))$ & 70.86 & 68.47 & 74.7 \\
\hline Average loading-bearing rate $\left(R_{a}(\mathrm{~N} / \mathrm{g})\right)$ & 71.34 & & \\
\hline
\end{tabular}

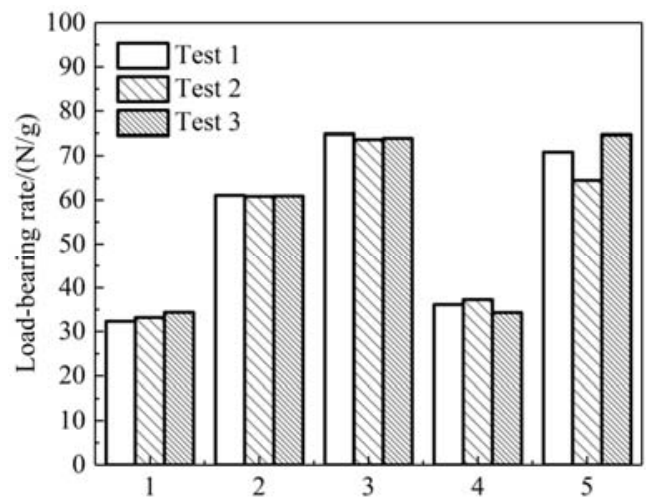

Figure 7. Bar chart of load-bearing capacity. 1: OS; 2: TOS; 3: TLNOS; 4: TLOS; 5: TLNIS.

\section{Conclusions}

To improve the load-bearing rate of the spacecraft rib, a structure-material integrated design framework has been proposed. The framework is based on the structure-material integrated design theory and is achieved using the commercial software Altair Solidthinking Inspire. Five types of optimized structures are fabricated by $3 \mathrm{D}$ printing and followed the tensile tests to obtained the loading-bearing rate. Results show that the structure-material integrated design framework can enhance the spacecraft rib's load-bearing rate by $122.73 \%$. TOS and TLNOS can increase the load-bearing rate by $82.97 \%$ and $21.73 \%$, respectively, compared with OS; while TLOS and TLNIS have increased the load-bearing rate by $7.78 \%$ and $114.23 \%$ respectively. These four structures are all less than $122.73 \%$, demonstrating the structure-material integrated design framework's effectiveness when designing the spacecraft rib. However, considering the aircraft's actual service situation, when designing the spacecraft rib, it is necessary to consider further the constraints of fatigue, aerodynamic load, and uncertainty [32-34] in the future study.

\section{Acknowledgements}

This research was financially supported by the National Natural Science Foundation of China (No. 11902085), and the Open Fund Project for Engineering Vehicle Lightweight and Reliable Technology Laboratory of Hunan Province (No. 2020YB04).

\section{References}

[1] Zhu, J. H., Zhang, W. H., \& Xia, L. (2016). Topology optimization in aircraft and aerospace structures design. Archives of Computational Methods in Engineering, 23 (4), 595-622.

[2] Bendsøe, M. P., \& Kikuchi, N. (1988). Generating optimal topologies in structural design using a homogenization method. Computer Methods in Applied Mechanics and Engineering, $71(2), 197-224$.

[3] Bendsøe, M. P., \& Sigmund, O. (1999). Material interpolation schemes in topology optimization. Archive of Applied Mechanics, 69 (9), 635-654.

[4] Xie, Y. M., \& Steven, G. P. (1993). A simple evolutionary procedure for structural optimization. Computers \& Structures, 49 (5), 885-896.

[5] Huang, X., \& Xie, Y. M. (2007). Convergent and mesh-independent solutions for the bi-directional evolutionary structural optimization method. Finite Elements in Analysis and Design, 43 (14), 1039-1049. 
[6] Wei, P., Li, Z., Li, X., \& Wang, M. Y. (2018). An 88-line MATLAB code for the parameterized level set method based topology optimization using radial basis functions. Structural and Multidisciplinary Optimization, 58 (2), 831-849.

[7] Cai, S., \& Zhang, W. (2020). An adaptive bubble method for structural shape and topology optimization. Computer Methods in Applied Mechanics and Engineering, 360, 112778 .

[8] Guo, X., Zhang, W., \& Zhong, W. (2014). Doing topology optimization explicitly and geometrically - a new moving morphable components based framework. Journal of Applied Mechanics, 81 (8).

[9] https://www.sohu.com/a/359727812_722157.

[10] Li, H., Luo, Z., Gao, L., \& Qin, Q. (2018). Topology optimization for concurrent design of structures with multi-patch microstructures by level sets. Computer Methods in Applied Mechanics and Engineering, 331, 536-561.

[11] Liu, J., Chen, T., Zhang, Y., Wen, G., Qing, Q., Wang, H.,... $\&$ Xie, Y. M. (2019). On sound insulation of pyramidal lattice sandwich structure. Composite Structures, 208, 385-394.

[12] Teimouri, M., Mahbod, M., \& Asgari, M. (2021, February). Topology-optimized hybrid solid-lattice structures for efficient mechanical performance. In Structures (Vol. 29, pp. 549-560). Elsevier.

[13] Zhang, J., \& Yanagimoto, J. (2021). Topology optimization of microlattice dome with enhanced stiffness and energy absorption for additive manufacturing. Composite Structures, $255,112889$.

[14] Wang, Z. P., Turteltaub, S., \& Abdalla, M. (2017). Shape optimization and optimal control for transient heat conduction problems using an isogeometric approach. Computers \& Structures, $185,59-74$.

[15] Wen, G., Ou, H., \& Liu, J. (2020). Ultra-wide band gap in a two-dimensional phononic crystal with hexagonal lattices. Materials Today Communications, 24, 100977.

[16] Yan, X., Huang, X., Zha, Y., \& Xie, Y. M. (2014). Concurrent topology optimization of structures and their composite microstructures. Computers \& Structures, 133, 103-110.

[17] Sivapuram, R., Dunning, P. D., \& Kim, H. A. (2016). Simultaneous material and structural optimization by multiscale topology optimization. Structural and multidisciplinary optimization, 54 (5), 1267-1281.

[18] Kazemi, H., Vaziri, A., \& Norato, J. A. (2020). Multi-material topology optimization of lattice structures using geometry projection. Computer Methods in Applied Mechanics and Engineering, 363, 112895.

[19] Wang, Y., Xu, H., \& Pasini, D. (2017). Multiscale isogeometric topology optimization for lattice materials. Computer Methods in Applied Mechanics and Engineering, 316, 568-585.

[20] Remouchamps, A., Bruyneel, M., Fleury, C., \& Grihon, S. (2011). Application of a bi-level scheme including topology optimization to the design of an aircraft pylon. Structural and Multidisciplinary Optimization, 44 (6), 739-750.
[21] Wong, J., Ryan, L., \& Kim, I. Y. (2018). Design optimization of aircraft landing gear assembly under dynamic loading. Structural and Multidisciplinary Optimization, 57 (3), 1357-1375.

[22] Félix, L., Gomes, A. A., \& Suleman, A. (2020). Topology optimization of the internal structure of an aircraft wing subjected to self-weight load. Engineering Optimization, 52 (7), 1119-1135.

[23] Liu, J., Ou, H., He, J., \& Wen, G. (2019). Topological design of a lightweight sandwich aircraft spoiler. Materials, 12 (19), 3225 .

[24] Wu, J., Clausen, A., \& Sigmund, O. (2017). Minimum compliance topology optimization of shell-infill composites for additive manufacturing. Computer Methods in Applied Mechanics and Engineering, 326, 358-375.

[25] Yu, H., Huang, J., Zou, B., Shao, W., \& Liu, J. (2020). Stress-constrained shell-lattice infill structural optimisation for additive manufacturing. Virtual and Physical Prototyping, 15 (1), 35-48.

[26] Qiu, W., Jin, P., Jin, S., Wang, C., Xia, L., Zhu, J., \& Shi, T. (2020). An evolutionary design approach to shell-infill structures. Additive Manufacturing, 34, 101382.

[27] Svanberg, K. (1987). The method of moving asymptotes-a new method for structural optimization. International Journal for Numerical Methods in Engineering, 24 (2), 359-373.

[28] Wang, H., Liu, J., \& Wen, G. (2020). An efficient evolutionary structural optimization method for multi-resolution designs. Structural and Multidisciplinary Optimization, 62 (2), 787-803.

[29] Long, K., Gu, C., Wang, X., Liu, J., Du, Y., Chen, Z., \& Saeed, N. (2019). A novel minimum weight formulation of topology optimization implemented with reanalysis approach. International Journal for Numerical Methods in Engineering, $120(5), 567-579$.

[30] Sigmund, O. (2001). A 99 line topology optimization code written in Matlab. Structural and Multidisciplinary Optimization, 21 (2), 120-127.

[31] Yi, B., Zhou, Y., Yoon, G. H., \& Saitou, K. (2019). Topology optimization of functionally-graded lattice structures with buckling constraints. Computer Methods in Applied Mechanics and Engineering, 354, 593-619.

[32] Chen, Z., Long, K., Wen, P., \& Nouman, S. (2020). Fatigue-resistance topology optimization of continuum structure by penalizing the cumulative fatigue damage. Advances in Engineering Software, 150, 102924.

[33] Gomes, P., \& Palacios, R. (2020). Aerodynamic-driven topology optimization of compliant airfoils. Structural and Multidisciplinary Optimization, 62, 2117-2130.

[34] Liu, J., Wen, G., \& Xie, Y. M. (2016). Layout optimization of continuum structures considering the probabilistic and fuzzy directional uncertainty of applied loads based on the cloud model. Structural and Multidisciplinary Optimization, 53 (1), 81-100. 